\title{
EL CARDENAL ALEJANDRO FARNESE EN LA CORTE PONTIFICIA DE RODRIGO BORJA Y LORENZO DE MEDICIS
}

\author{
Josep Maria LLORENS CISTERÓ
}

\section{Precedente}

El culto que el Renacimiento italiano tributó al “individuo" explica la idiosincrasia de la juventud aristotélica de aquella época. El ideal lo constituía el doncel a cuya belleza física acompañaba una preclara inteligencia, culto en filosofía neoplatónica y en artes liberales. Eran tan necesarias estas cualidades que, sin ellas el cortesano ni siquiera intentaba probar suerte en el campo de la celebridad.

El joven Alejandro, de vetusta prosapia y distinguida nobleza, pronto, por sus relevantes dotes naturales, excitó en su madre, doña Giovanella Caetani, el anhelo de ver elevada la Casa Farnese hasta la dignidad más suprema: el solio pontificio. Movida por tal aspiración, y para que los dones de la naturaleza alcanzasen completo desarrollo en su hijo, confió éste a los maestros más insignes del tiempo y al trato personal del duque Lorenzo de Medicis, portaestandarte del más acendrado fervor humanístico.

Pomponio Leto, famoso latinista llamado "Pontifex Maximus" de la Academia Romana, fue su primer preceptor. Empapado por éste en sólida cultura romana y expedito en la lengua del Lacio, Alejandro cumplidos sus veinte años, pasó a Florencia para perfeccionar sus estudios en aquella famosísima Universidad. Asentado en ella, cúpole en suerte ser huésped predilecto del singular mecenas Lorenzo de Medicis. Este encuentro representaría, sin duda, el factor decisivo en la orientación futura del joven soñador Farnese. En la Academia Medicea señoreaba el neoplatonismo. Los profesores Marsilio Ficino y Poliziano —-según afirma Rómulo Amaseivieron en el alumno Farnese a uno de sus más aventajados discípulos. Sus compañeros de estudio y amigos Pico della Mirandola y Giovanni de Medicis, el futuro Papa León X, le estimularon en el conocimiento de las bellas artes. Sin embargo, nadie como el propio Lorenzo ejerció sobre él acción más personal y decisiva. Una figura tan desconcertante, curiosa y polifacética como la de 
su mecenas que hablaba de poesía y literatura, que componía sonetos y chansons, que amaba la caza, que embaucaba al pueblo con grandes espectáculos, que no dudaba en echar mano de toda clase de medios para defender sus propios intereses, debía forzosamente impresionar el ánimo ávido de gloria del futuro Papa Paulo III.

En aquella corte, centro de artistas y literatos célebres, el joven Farnese trató a los insignes San Gallo y Michelangelo, a los que, cuando Cardenal, encomendaría la construcción de su palacio en Roma, y luego, como Papa, el ornato de la ciudad. También y sobre todo, pudo allí deleitarse en la audición de una de las Capillas de Música más famosas del tiempo. El neerlandés Henricus Isaac actuaba de maestro, sucesor del preclaro Squarcialupi muerto en el año 1475. Isaac conocido por Arrigo, Alamanus o Il Tedesco fue el preceptor de los hijos de Lorenzo y del huésped Farnese.

En un inventario de los objetos de palacio realizado durante el mandato del duque Lorenzo, se registran cinco órganos, de los cuales tres eran "positivos" y dos "portátiles"; además de varios ejemplares de los restantes instrumentos en uso y unos pocos de tradición más antigua'. Se sabe que el órgano era el instrumento predilecto de tan ilustre persona, en cuya práctica frecuentemente se ejercitaba. Una corte en fin, que en comunión con el platonismo, vivía intensamente un clima musical promovido por su señor el duque Lorenzo de Medicis².

\section{Alejandro Farnese en Roma}

Al término de su permanencia en la ciudad de Florencia, Alejandro Farnese estaba bien preparado para prometerse una brillante carrera en la Corte Pontificia, cuya meta habría de ser la tiara.

Con el fin de iniciar esta nueva etapa de su vida, el día 10 de abril de 1489 emprende viaje a Roma. La primera recomendación la obtuvo del mismo Magnífico a tenor de la siguiente carta dirigida a su embajador en Roma, Giovanni Lanfredini:

"Io vi scripsi a di passati in favore di messer Alexandro da Farnese et al presente ha facto pensiero venire di costà, forse per la medesima cagione, et per essere conosciuto dalla Santità di N.S. per suo fedelisimo servitore. Io voglio farve intender che costui, oltre allo essere nato della casa che è, ha molte et singolari parte in sè, tra le quali sono molto abundante le lettere et buoni costumi perchè è et doctissimo et uno exemplo di buona et laudabile vita. Per questa cagione, che sapete quanto mi sogliono muovere, velo raccomando quanto farei mio figluolo, et vi priegho lo introduciate et raccomandiate caldassimamente a N.S. Di che non potresti farmi maggiore piacere, ne io potrei esserne più contento. Florentiae die X aprilis 1489. Laurentius de Medicis." 3

Esta carta iba acompañada de una nota que dice:

1. J. Wolf: "Isaac in Firenze", La nuova musica, Florencia, 1907.

2. F. GHisi: I Canti Carnacialeschi nelle Fonti Musicali del XV e XVI secolo, Florencia-Roma, 1937; Feste musicali della Firenze medicea (1480-1589), Florencia, 1939.

3. Archivio di Stato, Firenze. Carteggio Mediceo innanzi il Principato, filza XXXIX, n 594. 


\footnotetext{
"Mandovi in questa una informatione de uno de quest Signori da Farnese che si trova a studiare, a mio parere molto gentile giovane, litteratissimo et costumato. Prieghovi facciate ogni forza di servirlo". 4
}

Cuando más adelante, se le propuso para el cargo de secretario de cancillería, poco inclinado todavía a recibir las órdenes sagradas, fue el propio Lorenzo quien le obtuvo la dispensa oportuna de vestir el hábito y roquete que competía a todo protonotario apostólico. Esta nueva intervención del duque a favor de Farnese subraya el aprecio que le seguía prodigando a distancia de tiempo y lugar 5 .

\section{El cardenal Alejandro Farnese}

Alejandro Farnese, el día 20 de septiembre de 1493, a los veintiún años de edad, recibía el capello cardenalicio. En buena sazón iniciaba la etapa más importante de su carrera diplomática. Príncipe de la Iglesia, imbuído también, aunque posiblemente en menor grado, del espíritu mundano que favorecía el Renacimiento, recibió las órdenes menores, el subdiaconado y el diaconado. Su prestigio aumenta en los pontificados de Julio II, León X y Clemente VII, hasta sucederle en el gobierno de la Iglesia. Julio II, le transfirió el título de S. Eustaquio, más lucrativo que el que gozaba de los Santos Cosme y Damián. Además, mediante bulas del 8 de julio de 1505, declaró legítimos a sus dos hijos Pierluigi y Paolo, que había engendrado para no dejar sin sucesión a la familia Farnese, por ser él el único vástago varón.

Los Cardenales vivían con todo el boato propio de una corte principesca. Las pingües y numerosas rentas que percibían les permitían convertir su palacio en un cenáculo de artistas y literatos, según el gusto de cada uno de ellos. En el censo que se hizo en Roma, año 1526, se describe el número exacto de las personas que componían la familia de los cardenales. Por la elevada cifra que arroja, deducimos el rango de los mismos. La del Cardenal Farnese contaba con 306 domiciliados. Era la más numerosa, a la que sólo aventajaba la familia del Papa Clemente VII que constaba de 700 miembros $^{6}$.

Paolo Cortese, en su obra "De Cardinalatu" nos ofrece datos interesantísimos sobre la vida y costumbres de los purpurados. Cuando precisa las cualidades acústicas que debe poseer el lugar dispuesto para la audición de música, observa:

\footnotetext{
"Quod cubiculum musicae debet esse fornicatum et rotundum propter vocem, et debet habere vasa musica in pariete collocata ad consonantiam".
}

Contra quienes consideran la música como inútil y perniciosa, sostiene:

\footnotetext{
4. Ibidem, $\mathrm{n}^{\circ} 589$.

5. Ibídem, $\mathrm{n}^{\circ} 590$

1894.

6. Domenico Gnoli: Descriptio Urbis, O Censimento della Popolazione di Roma avanti il Saco borbonico, Roma,

7. Paolo Cortese: De Cardinalatu (1510), Liber II, f. LI.
}

[3] 
"Non modo delectationis, sed etiam disciplinae morumque causa".

Y sigue:

"Intelligendum est senatores debere in sonantium genere audiendo versari".

Más adelante distingue tres modos de cantar, y señala la diferencia de gusto existente entre los cantores franceses y españoles de la Corte pontificia:

"Phrygia enim est qua animi audientium acriori vocum contentione abalienari solent: ex quo genere illa numeratur qua gallici musici in palatino sacello natalitiis ex suscitatisque feriis rituali lege utuntur: lydia autem duplex indicari potest una quae coagmentata, altera quae simplex nominatur: coagmentata enim est, quo in flexo ad dolorem modo animi ad fletum misericordiamque deducuntur: qualis ea videre potest quo novendilia pontificia, aut senatoria parentalia celebrari solent: quo quidem lugubri canendi genere semper est natio hispanorum usa".

Todavía sobre el mismo tema, insiste Baldesar Castiglione ${ }^{9}$ :

"Non mi contento del cortegiano, s'egli non è ancor musier, e se oltre allo intendere ed essere sicuro a libro, non sa di varii strumenti"; “...esser la musica non solamente ornamento, ma necessaria al Cortegiano"; "Dà ornamento e grazia assai la voce umana a tutti questi instrumenti de'quali voglio che el nostro Cortegian basti aver notizia, e quanto più però sarà eccelente, tanto sarà meglio".

Consta de muchos cardenales que conocían bien la teoría musical, que gustaban de discutir sobre ella, y que se deleitaban tocando algunos instrumentos. El propio Farnese retuvo durante ocho meses el tratado de Música de Ptolomeo que le fue prestado por el bibliotecario de la Vaticana Monseñor Pedro Garsía, obispo de Barcelona ${ }^{10}$. Ippólito de Medicis tocaba el violín, la flauta y el laúd ${ }^{11}$.

Según el relator Marín Zorzi, el cardenal Grimani:

"era docto in humanità e jure canonico e sopra tutto musicho excellentissimo; et quando el canta con qualche uno li fa donar 100 e più ducati". 12

Fiestas, recepciones, banquetes y ceremonias llenaban en gran parte la vida cortesana de los cardenales. Es ésta una de las notas más relevantes de la acuciante actividad del espíritu en un siglo de fundamental importancia en el desarrollo y plasmación del carácter italiano. Diarios y crónicas nos describen minuciosamente el clima de tales reuniones. La etiqueta era nota distintiva de cada corte, y la música jugaba su importante papel artístico y funcional. Para hacerse con la idea, transcribo solamente el desarrollo de un banquete: el ofrecido en 1505 por el cardenal Grimani a los enviados de la República Veneciana, presente el Cardenal Farnese. Por lo prolijo

\footnotetext{
8. Ibídem, f. LXXIIv.

9. Baldesar CASTIGlione: Il Cortigiano, ed. V. Cian, Florencia, 1894, Libr. I, 101, 106; Libr. II, 139.

10. Biblioteca Vaticana, Vaticano Latino 3966, f.3v.

11. B. Amante: Giulia Gonzaga, Bolonia, 1896, 85 ss.

12. Marino Sanuto: I Diarii, XXIV, col. 93, 17 marzo de 1517.
} 
de la narración, me limitaré únicamente a su parte musical ${ }^{13}$. A las puertas del Palacio Grimani, un nutrido conjunto de trompetas, pífanos y tamburiles tributaban el saludo de bienvenida a los distinguidos huéspedes. Ya dentro, y antes de tomar asiento, se les ofrecía para las manos agua perfumada. Dispuesta la comida, Giovanni da Bologna, camarero secreto del Cardenal acompañado de todos los servidores, desfilaron al son de un buen grupo de pífanos y trompetas. Luego fueron presentados los siguientes platos:

\begin{abstract}
"Confetione damaschine [...] con fiori e ruose molto galante. Poi 18 confetione d'oro e d'argento con 74 pignochate dorate e suoi biscotelli, accompagnate con "tamburini et arpe".

Poi capi di late con zucharo et aqua ruosa in taze a tutti li oratori [...] acompagnati da una suave musicha. Poi 18 piati di suppe de duca, con animelle e teste di capreto dorate, zaschun con la sua bandirola d'oro con San Marcho et arme del Cardinale, acompagnate a son di trombe squarzate. Poi 74 taze di pollastri fatti di arosto menuto, a quaie 10, pizoni 6 e polaster 6 per piatto con naranze [...] vin San Severin dolze, acompagnati con un altra excelente musica la qual durò fin al levar di pastizi menuti in quazero, che foron portati da poi lo arosto menuto. Poi 18 piati di arosto, a doi fasani e uno favon per piato, tutti vestiti al collo e la coda de suo pene et il petto a tutto dosato, con menestra, fior de zanestra e sapor salsa reale acompagnati con certa sorte de buffoni, che di persona, bocha, ochii, naso et atti si contrafeva et filava et feva molte altre bufonerie da rider. Poi 18 piati di miraustro a 8 pizoni per piato con naranze dolce e vin bianco brusco accompagnati da dui buffoni spagnuoli con zimbali d'argento in man, che cantavano a l'improviso". Poi 18 di alesso [...] con arpe e viole. Poi 18 piati di caponi coperti [...] acompagnati da un buffon albanese che si chiama Barleta, vestito tutto d'oro con uno suo tamburo fornito tutto d'argento et uno compagno con una violeta che sonaron alcuni canti soavi, dellicati et molto degni. Poi piati 18 de porcho zengiaro, caprioli et lepori [...] acompagnate con arpe et violete. Poi taze 64 de zeladina dorata e vin Corso asutto, con un'altra musica non men suave e degna de le altre. Poi racota batuta [...] con dui putti vestiti da pastori recitando alcuni versi in laude di la illustrissima Signoria di reverendissimo Cardinale e di prefati Oratori. Poi 18 marzapani in 18 piatti [...] acompagnati con una zentil moresca ballata con somma galanteria. Poi levato uno mantile fu data aqua rosa lavanda a le mane a son de pifferi, trombetti et tamburini, e venne confetti [...] con Jebia e due compagni che sonaron due viole grande da archeto con grandissima suavità et gratitudine di tutti."
\end{abstract}

En 1513, para solemnizar el título de ciudadanía romana que se concedió a Giuliano de Medicis, hermano de León X y a toda su iamilia, se celebró en la Iglesia d'Aracoeli una solemne misa pontifical en la que tomaron parte los cantores del Papa. Después siguió un festín en el cual se recitaron versos con exquisita música ${ }^{14}$.

\title{
Alejandro Farnese en la Corte de Rodrigo Borja
}

Las experiencias vividas en la fastuosa y desconcertante Corte de los Borja habrían de continuar grabando en el ánimo de Farnese las impresiones recibidas en la Corte de los Medicis. Sin entrar en detalles, y a manera de síntesis, se recuerda que Alejandro VI no era insensible al

13. Ibídem, VI, col. 171, ano 1505

14. Domenico GNoli: "Il Teatro Capitolino", Nuova Antologia, Roma, 1930. 
deleite del espíritu, Tuvo sus músicos, poetas, oradores y literatos. Su entretenimiento favorito, se sabe, fue el teatro. El español Juan del Encina, le preparaba con frecuencia representaciones que aquellos nobles dignatarios hallaban deliciosas. Por sus cualidades de poeta, músico y compositor, Juan del Encina supo entreverar todos estos elementos para lograr una única realidad estética, rica en matices y colorido artístico ${ }^{15}$.

En los pocos registros conservados de este Pontífice, sólo he visto anotadas pagas de esta índole:

"Bon .50 pro libris decem turronum pro cantoribus, ad bon.5 por libra" Bon. 27 pro tribus libris confectionum pro colatione cantorum, cum scatulis". 16

Acostumbraba, además, el Papa satisfacer mensualmente honorarios de seis escudos para cada uno de los dos tubicines que prestaban servicio a Alfonso de Aragón y para los que acompanaban a su sobrino Rodrigo. También, en atención a los florentinos que solemnizaban la fiesta de San Juan, permitía que los trombetti i pifferi di Castello (S. Angelo) actuasen en el desfile de carrozas alegóricas ${ }^{17}$. A los pocos días de ser coronado Pontífice, septiembre de 1492, el Camarlengo anota los nombres de los siguientes cantores que habitualmente prestaban servicio ${ }^{18}$ :

\begin{abstract}
"R. Sancti Georgii etc [...] solvi faciatis infrascriptis officialibus et cantoribus Capellae S.D.N. sumam pecuniarum infrascriptam pro eorum provisione mensis septembris proximi preteriti et primo Rdo. in Cristo domino Episcopo Barchinonensis Magistro Capellae, florenos X. Rdo. in Cristo domino Abbati Sancti Sebastiani Sacristae Capellae florenos X. Jo. Mostruel - Jo. Radulphi - Ar. Blasii - Re. de Mastagnis - En. de Havresse - Ant. Banistori - Inno Cosee - Jo. de Meruen - Bertr. Vaqueras - Mar. de Orto - Pe. Lefranch - Crist. Rousseau - Jo. Barbe - Jo. Baltassar - Geor. de Dunno - Judo de Spres (Desprez) - Jo. Juvenis - Reg. de Honderie - Ph. de Prunis - Ro. de Anglia - Jo. de Aragonia - Jo. de Lanas (Illanas)."
\end{abstract}

Cada uno de ellos recibe 8 florines mensuales. Sucesivamente el mismo Camerlengo va repitiendo por meses los nombres de los componentes de la Capilla, y señalando los cambios y modificaciones. Así, en octubre añade a Donato Felix y a Jo. de Verona a la vez que omite a Jo. de Aragón. La laguna en los registros en cuestión, que abarca de abril de 1494 al año 1500 inclusive, explica la carencia de noticias. Los libros complementarios Introitus et Exitus ${ }^{19}$ nada especifican con la lacónica fórmula "Cantoribus et Officialibus Capellae Sanctissimi", con la asignación global por meses concedida a los cantores que, oscilando entre los 235 y 311 escudos, se entregaban al decano del Colegio, Remigio de Mastagne. A partir de febrero de 1501 aparecen de nuevo los cantores anotados por su nombre, según el orden siguiente ${ }^{20}$ :

15. R. Stevenson: "Spanish musical impact beyond the Pyrenees (1250-1500), Actas del Congreso: "España en la Música de Occidente", Salamanca, 1978, I, 155-161; R. SHERr: "A Note on the Biography of Juan del Encina", Bulletin of the Comediantes, XXXIV/2, Winter, 1982, 168. 1503. Pascha.

16. Archivio Secreto Vaticano, Libro delle minute spesse di Palazzo, Die 24 decembris, 1502 ; Die 16 aprilis,

17. Mario Menottr: Documenti inediti sulla famiglia e la corte di Alessandro VI. Roma, 1917, 212

18. ArChivio di Stato, Roma, Archivio della R.C.A. Parte prima, Mandati Camerali, 855, ff. 3, 4, 7, 11, 35, 41, 49, $55,72,76,79,84,85,94,102,106$.

19. Archivio Secreto Vaticano, Introitus et Exitus, 526-534.

20. Archivio di Stato, Roma, Archivio della R.C.A. Parte prima Mandati Camerali, 858, ff. 9, 19, 28, 36, 43, 51 , $59,65,73,83,89,97,103,109,117$. 


\begin{abstract}
"Antonio Barreston - Bertrando Vacqueras - Xrosforo Rouseau - Jo. Barbo - Jo. Le petit (borrado) - Philippo de Prunnis - Jo. de Illanas - Donato Felice - Jeronimo Bertrandi - Crispino de Stapen Augustino de Nigris - Jacobo Vulgot - Antonio Waltheri - Alfonso de Troia - Paulo de Trottis - Martiriano (Prats) - Gaspare Werberek - Bon. Radulphi - Jo. Gruter.
\end{abstract}

En abril viene añadido Iohannes Baltazar, mientras en enero de 1502 desaparecen Bertrando Vacqueras y Agostino de Nigris. El toledano Alfonso de Frias, García Salinas y Iohannes Pocquetoy entran en febrero del mismo año.

Finalmente, la relación de los cantores beneficiarios de la distribución del paño negro, con motivo de los funerales del sucesor de Alejandro VI (Papa Pío III, cuyo pontificado duró solamente 26 días, del 22 de septiembre a 18 de octubre) menciona los siguientes nombres ${ }^{21}$ :

\footnotetext{
"Johannes de Meruen - Bertrandus Vacqueras - Cristophorus Rousseau - Philippus de Prunnis Johannes de Lyanas - Crispinus de Stappen - Jacobus Valpot - Antonius Vulterii - Paulus de Trottis - Gaspar Werbeck - Bon. Radulphi - Iohannes Grutter - Alfonsus Frias - Garsias Salinas - Marturianus Prats - Thomas Jacobi - Johannes Pocquetoy - Johannes Scribanus - Johannes Palomares".
}

A los cantores hemos de añadir a "Bernardino Asculo cum duobus sociis tibicinibus" y a "Alexandra de Pisis in musica perita" los cuales recibían su habitual remuneración, así como "Johannes Orfani scriptor". Alejandro VI, además de haber aumentado el número de cantores españoles conferió mediante Motu propio el cargo de Maestro de Capilla al Bibliotecario Mons. Pedro Garsia, Obispo de Barcelona (1503) primero, y a Bartolomé Martín, Obispo de Segorbe después ${ }^{22}$.

Sobre algunas actuaciones de la Capilla durante el pontificado de Alejandro VI, cabe recordar la información que da el Maestro de ceremonias Johannes Burckardus, con referencia al canto de la Pasión del Domingo de Ramos del año de $1499^{23}$ :

\begin{abstract}
"Passionem dixerunt tres hispani, quidam novus senex in voce evangelistae, alius cantor capellae in voce judei et d. Raphael de Arena, diaconus capellae in voce Christi. Bene vociferati erant omnes, et si accentus et cantum capellae observassent simpliciter, cantassent optimae, sed ubi morem Hispaniarum miscebant nostro, male sonabat. Verba flevit amare, emisit spiritum, contra sepulchrum cantaverunt omnes tribus vocibus suavissime".
\end{abstract}

A continuación, con referencia a las lecciones del oficio de Tinieblas del Miércoles Santo, explica el mismo Burckardo:

"Ubi est prima, secundam lectionem sive tertiam cantaverunt soli hispani per quatuor voces laudabiliter".

21. Archivio Secreto Vaticano, Vaticano Latino, 9027, ff. 163-169.

22. Conrado Eubel: Hierarchia Catholica Medii Aevi, Münster, 1914, 102, 234. Burchardus refiere que el Sábado in Albis de 1493, Pedro obispo de Barcelona, maestro de la capilla del papa, ofició de pontifical en el Palacio Apostólico.

23. Iohannis Burchardi, Liber Notarum a cura di Enrico Celani, Città di Castello, 1911, 132. Véase también M. SCHULER: "Spanische Musikeinflüsse in Roma um 1500”, Anuario Musical XXV, Barcelona, 1970, 28-32. 
Y en las del Viernes Santo añade:

"Iidem Hispani cantarunt, dicta secunda lectione finita, primam, secundam et tertiam lectionem in Matutinis in basilica Sancti Petri cum magna omnium laude et gratia".

Como favores espirituales concedidos por el Pontífice, Burckardo señala la facultad otorgada a los confesores elegidos por cada uno de los cantores, de perdonarles todos los pecados con indulgencia plenaria, durante la cuaresma.

En el año 1500, unos días antes del Jubileo quiso el Papa que ensayasen en su presencia el motete que habían compuesto para la apertura de la Puerta Santa, "et auditu ipsum laudavit" Ante tan variados y frecuentes acontecimientos familiares del Papa Borja, es fácil suponer la actuación en palacio de una capilla musical privada compuesta de cantores e instrumentistas. La laguna, con todo, de los registros de la Tesorería Secreta de este pontificado, no permite ofrecer datos oficiales sobre ello. Para suplir. en lo que cabe esta laguna, se pueden aducir algunas referencias sobre los festejos nupciales de su hija Lucrecia. En efecto, cuando ésta casó con Giovanni Sforza de Pésaro, el banquete celebrado en el Vaticano, presidido por el Papa con su acompañamiento de doce cardenales, terminó con danzas, cantos y músicas.

Del segundo matrimonio con Alfonso I d'Este sabemos más pormenores. Nicolò Cagnolo, testigo ocular, refiere que el día 2 de febrero de 1502, la esposa Lucrecia, hizo su entrada triunfal en la ciudad de Ferrara al son de un conjunto musical compuesto por ochenta y seis trompetas y muchísimos pífanos. El espectáculo resultó singular en su tiempo. Por espacio de seis días se sucedieron en aquella corte las tres diversiones favoritas: banquetes, danzas y representaciones teatrales. En aquel entonces, los festejos fácilmente derivaban hacia lo grotesco. El teatro constituía el pasatiempo preferido y más peculiar de la ciudad de Ferrara. Ello explica que el duque pusiera singular empeño en la selección de aquellas representaciones que habían de satisfacer plenamente las exigencias de un tan abigarrado conjunto de espectadores, de atuendo variado y polícromo. En resumen, la parte musical consistía en interpretar danzas "alla moda Romanesca e Spagnuola con tamburini". También en la interpolación de cantos, recitativos y piezas instrumentales. Las comedias más aplaudidas fueron las de Plauto, en particular: Epídico, Bacchides, Asinaria y Cassina. En el desarrollo de las mismas hallamos las siguientes indicaciones ${ }^{25}$ :

\footnotetext{
"suoni, canti e melodie de diverse sorte, laudabili e dilettevoli. Sopraggiunsero otto cantori, fra quali era una femina di Mantova con tre liuti, qualli mellifluamente cantarono una barzelleta con grandissima arte in tre stanze e subito fu cominciata la $3^{\mathrm{a}}$ parte della comedia e subito fu udita un'armonia di zufoli (flauta campestre) con grande eleganza".
}

24. Liber Notarum, 90, 64, 264. ¿Sería tal vez el Salve regis mater sanctissima? (Cap. Sist. 35, ff. 188v-191): Véase J.M. Llorens: "La Música a capella en la Capilla pontificia durante los pontificados anteriores a Paulo III", Revista de Musicología (SEM), VI, 1983, $\mathrm{n}^{\circ} 1-2,309-326$.

25. F. Gregorovius: Geschichte der Stad Rom im Mittelalter XIII, edic. W. Kampf, Darmstadt, 1957; F. Gregorovius: Lucrecia Borja según los documentos y correspondencias de su propio tiempo, trad. A. Escarpizo. Editorial Lorenzana, 1962 , 302-317. 
En la comedia Cassina se precisa:

"Due musiche interposte cantate artifiziosamente a con dolci armonie che furono cantilene in lode delli Illmi. D. Alfonso e Madama Lucrezia, sposi, con alcune moresche fra quali venne fuori un uomo selvatico con un corno, sonando con una bella damigella che il seguitava con altri uomini selvatici ballando alla moresca tutti degnamente e subito ivi apparve il Dio d'Amore che scacciava e percote $v$ a detti uomini selvatici, accompagnato da certi musici, quali tolsero detta damigella in mezzo cantando soavemente; poi ad un altro istante venne fuori una grossisima palla quale in mezzo del poscenio se aperse in due e ivi entro c'era una bellissima musica con lire ed armonie soavi cantando".

En esta ocasión la hermana del duque, Isabella d'Este, marquesa de Mantova llamó la atención de todos al cantar maravillosamente bien, acompañándose con el laúd. Castiglione y Bembo en más de una ocasión la celebran por tales cualidades.

La intervención de las violas aparece frecuentemente en el relato de los actos celebrados. A veces, en grupo de seis. Parece ser que en Ferrara el arte de tocar las violas había alcanzado un grado tan notable de perfección que cuando César Borja salió en 1498 hacia la corte de Francia pidió al duque Hércules que le facilitase algunos de aquellos instrumentistas para llevarlos consigo, pues en aquel país los artistas ferrareses eran muy estimados. El texto de la carta de César Borja escribe: Violas arcu pulsantes ${ }^{26}$.

De las fiestas religiosas sólo se menciona la celebrada el domingo, día 6 de febrero, en la catedral, cuya narración dice:

"verso il Duomo con tutti li gentiluomini a due a due davante e molti trombetti che suonavanno,
e intratti nella Chiesa Maggiore, si cominció una solennissima Messa con cantori e organi che
fu celebrata per un vescovo spagnuolo Cancelliere del Beatissimo Papa con gran ceremonie". 27

En consonancia con estas formas cortesanas, Doña Giovanella Caetani, madre de nuestro Alejandro, quiso celebrar en Capodimonte, residencia de los Farnese, la reciente elevación de su hijo a la púrpura. El Papa Alejandro VI aceptó la invitación. Este fue el primer ensayo del flamante cardenal en tal fausto acontecimiento ante un espléndido concurso de invitados.

\section{Alejandro Farnese y el Papa León X}

De los seis pontífices con quien convivió Alejandro Farnese en dignidad de cardenal, el que ejerció mayor influjo en la orientación de su gusto artístico y en la ostentación de vida palaciega fue, sin duda, Juan de Medicis, el Papa León X, íntimo amigo de juventud en Florencia. Cupo al Farnese - primer cardenal diácono- proclamar la elección de Medicis para la sede que había vacado por muerte de Alejandro VI. Merced a las innumerables pruebas de afecto con que le distinguió el Papa León X, la corte Farnese pudo sobresalir en magnificencia y esplendor. Por

26. F. GREgorovius: Lucrecia Borja, Barcelona, 1962, 313-318.

27. Niccolò Cagnolo de Parma que había acompanado al embajador francés para los festejos de Ferrara. Véase Cronica manoscrita del Zambotto en la Biblioteca de Ferrara. 
espacio de muchos años, el Cardenal Alejandro sintió la inquietud de procurarse un palacio que respondiese a su rango y grandeza y a su anhelo de acoger en él a los personajes más eruditos y a los artistas más famosos. A tal efecto inició la construcción del suntuoso edificio situado en la plaza homónima, en Roma.

La historia de las tres generaciones que endulzaron en él su vida, a saber, Alejandro Senior (Paulo III), Pierluigi su hijo, Alejandro Iunior y Odoardo, hijos de Pierluigi, llena uno de los capítulos más notables de la cultura renacentista del siglo XVI. León X fue un auténtico Papa humanista. Su pontificado ha sido estudiado por muchos historiadores que, dentro de sus múltiples diferencias, concuerdan en destacar la predilección suma que manifestó por la música y la estima que dispensó a sus músicos, prefiriéndolos a los demás artistas de su corte. Abunda la documentación que considera al Papa Medicis como hombre versado en música. En efecto, Pablo Cortese le reputaba ${ }^{28}$ :

"Homo in musicis litterata pervestigatione prudens".

Platina, en su biografía, afirma que:

"amó sumamente i musici, come colui ch'era molto in quell'arte dotto". ${ }^{29}$

Fabroni añade que tenía una voz melodiosa y agradable y que aprendió a cantar desde su infancia $^{30}$. Rafael Brandolino Lippo informa que siguió el ejemplo de su padre el duque Lorenzo Medicis, interesado porque siempre en su iglesia dedicada a San Juan en Florencia, se alabase al Señor $^{31}$ :

\footnotetext{
"suavissimo vocum concentu" y añade "et musicam artem tanto studio olim perdidicit ut nunc praestantissimis ejus doctoribus antecellat et sic ea successivis temporibus delectatur ut cum lectissimis cantoribus, ipse interim canat, canentes quandoque alios attentissime audiat, addibita non nunquam varia jucundaque fidium harmonia".
}

El mismo Brandolino en otro escrito ${ }^{32}$ repite que su protector el Papa León X se procura los músicos más competentes, instrumentistas y cantores con que dispone conciertos que son "honestissimum voluptatis genus". Pedro Martín d'Anguera escribía desde Valladolid, 20 de abril de 1513:

"Habemus Pontificem eruditum, sed musicum, et qui cantorum collegiis et frequenti corona delectatur". ${ }^{33}$

Sintió inclinación por todos los géneros musicales. Una carta de 30 de julio de 1501 , suplicada para el cardenal Giovanni de Medicis, de parte del Abad de Saint-Bertin, Antonio

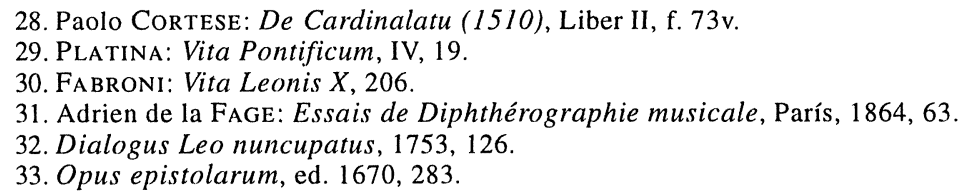


Berger, por mediación de Erasmo de Roterdam, nos informa que le fueron enviadas duas cantiunculas que espera serán de su $\operatorname{agrado}^{34}$ :

"vel quod est ipse (Iohannes Medicis) huius artis antiquissimae longe scientissimus, itidem ut aliarum omnium; vel quod cantilena recens est et nuperrime nata, et ex eo quidem nata qui se quondam in clarissima Medicum familia praedicat alitum fuisse; quae mihi nimirum ingeniis excitandis ornandisque studiis coelitus data fuisse videtur. Is est in nostra urbe musicae artis princeps".

Su peculiar predilección por los buenos músicos la ponen de relieve tanto Wilhelm Eysengreis en su Historia de Spira cuando escribe: "Leo decimus musicos in primis amavit", como Pietro Aaron en su Il Thoscanello della musica al decir que el Papa llamaba a numerosos artistas para servirle en su Corte pontificia con el estímulo de retribuirles con largueza. En este sentido, músicos de las cortes de Mantua, Ferrara, Florencia y otras incluso no italianas, pasaron a la pontificia. De ello nos dan pruebas las elevadas cantidades de dinero consignadas en los registros del Camarlengo y las prebendas conferidas. Ludovico Pastor ${ }^{36}$, J. Hergenröther ${ }^{37}$ y Herman-Walter Frey ${ }^{38}$ ofrecen listas bastante completas. Entre los más favorecidos señalemos a Esteban Gabriel Merino, nacido en San Esteban del Puerto (Jaen) el año 1472. Al quedar huérfano de padre,muy joven pasó al servicio del Cardena Ascanio Sforza, en Roma; León X, el 7 de mayo de 1513, le nombró Arzobispo de Bari, luego, el $1^{\circ}$ de mayo de 1514, le creó conde palatino y le confirió once beneficios eclesiásticos que le reportaban la pingüe renta anual de 700 escudos de oro. Mereció tales prebendas por sus servicios, habilidad diplomática y, en particular, por sus excelentes disposiciones musicales. Durante este pontificado, estuvo al frente de la Capilla musical privada con la misión de proveerla de buenos músicos ${ }^{39}$. También distinguió el Papa a Peñalosa ${ }^{40}$, otro español insigne, autor de composiciones profanas y religiosas; al judío Johannnes Maria Germanus $^{41}$ y a los dos maestros Nicolás de Pictis y Elziario Genet.

Siempre pródigo, el Papa recompensaba toda intervención musical en honor suyo. He aquí algunas referencias sacadas del registro de la Tesorería Secreta ${ }^{42}$ :

"E più a un pucto ferrarese sonò di bono accordo in Castello, duc. 20"

"E più a un pucto cantore di m.r. R.mo d'Aragona, duc. 2".

"E più a quello sonò la lira la Rocha di Viterbo, duc. 2".

34. P. S. Allen: Opus Epistolarum Desiderii Erasmi, 1906, 371.

35. Chronologicarum rerum amplissimaeque urbis Spirae libri XVI, 1564, f. 287.

36. Storia dei Papi, Roma IV, 1945, 378-379.

37. Leonis Pontificis Maximi Regesta, ed. Joseph Hergenröter, I-VIII, Freiburc i B: Herder, 1884-1891.

38. "Regesten zur päpstlichen Kapelle unter Leo X und zu seiner Privatkapelle", Die Musikforschung, VIII, 1955, 58-79, 178-179, 184-185, 412-439; IX, 46-57, 139-156.

39. A. Ferrajoli: "Il ruolo della Corte di Leone X. Prelati Domestici", Archivio della R. Società Romana di Storia Patria, XXXV, Roma 1912, 219. "Gabriele Spagnolo, detto Gabrielletto, nel tempo che fu da fanciullo fu veduto in Roma governare i cani in casa del Cardinale Ascanio, portar legna pel fuoco dell'Anticamera, di poi della camera di detto Cardinale, col qual mez:z: riusci sotto cameriero e finalmente cameriero di quel signore, et con gli anni tuttavia crescendo di autorità e di ricchezza, divennne arcivescovo di Bari, patriarca delle Indie e cardinale di Clemente VII, assai stimato dal Collegio et molto più da Carlo V Imperatore". Girolamo Garimberti: La prima parte delle vite di alcuni papi e cardinali. Venezia, 1567, 348.

40. D. Preciado: Francisco de Peñalosa (ca. 1470-1528) Opera Omnia. I Madrid 1986; II Madrid 1991.

41. Este y su hijo Camilo recibían además de cuantiosas pagas extraordinarias, la mensualidad de 23 escudos de oro.

42. ArChivio Di Stato, Roma, Spese private di Leone X, Johannes Lazarus de Magistris "Serapica", I, II, III. 
"E più uno putto trombettier e certe donne che cantavano, duc. 1".

"E più a uno sonava la cithara, duc 1 ".

"E più a li musici di Mons. R.mo d'Aragona, duc. 125".

"E 6 musici de Re di Francia vestiti di bianco, duc. 150".

"E li tre Franzesi cha fanno la moresca, duc. 63".

"A una donna Pistolese che canta, duc. 10".

"A tre sonatori de arpa, tamborino et violetta che sonarono el di de S. Ioanni innati a N.S., duc. 5".

Las lagunas que se echan de menos en los Mandati Camerali nos impiden seguir el movimiento de la plantilla de los cantores pontificios. La lista más próxima, 1 de marzo de 1509 , a la elección de León $\mathrm{X}$ anota los nombres siguientes ${ }^{43}$ :

"Solvatis infrascriptis dominis,

R.P.D. Francisco, episcopo Suessanensis, magistro dicte Capelle duc. auri decem.

R.P.D. Gabrieli Archiepiscopo Dirachiensis, duc. decem.

Remigio Masting

Gaspari Merbach (léese Werbeck)

Jo. de Ilianis

Jacobo Valpot

Paolo de Trottis

Alfonso Frias

Thome de Fazanis

Jo. Poqueton

Jo. Scribano

Jo. Palomares

Michaeli Touppe

Matheo de Alzate

Nicolai de Pittis

Jo. Radulphi

Elziario Genesi

Egidio Carpentier

Valentino Vnigaro

Cantoribus dicte Capelle duc. auri octo pro quolibet.

Johanni scriptori dicte capelle duc. similes duos.

Datum Rome in Camera Apostolica, die prima martii 1509,

pontificatus Sancmi. domini Nostri domini Julii pape secundi, anno sexto."

Asimismo, la relación de cantores más inmediata, 3 de septiembre de 1523, a la muerte del Papa, cita los nombres siguientes ${ }^{44}$ :
Paulus Trottus
Michael Clementis
Thomas de Fazanis
Joan. Franc. Felicis
Jo. Scribanus
Alexander Coppin
Jo. de Palomares

43. Archivio Di Stato, Roma, Mandati Camerali, $857, f .157 v$. En el f. $174 \mathrm{v}$ se libra un pagamento de 4 escudos a Raynerio cantore.

44. F. X. HABERL: Baustaine für Musikgeschichte III (Die römische "Scola Cantorum" und die päpstlichen Kapellsänger bis zur Mitte des 16 Janrhunderts) 259. 


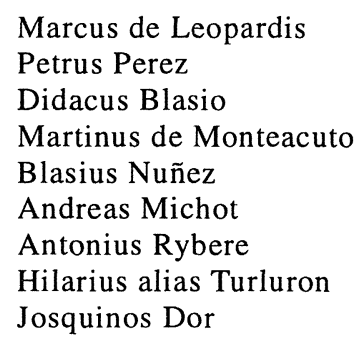

Entre estas dos relaciones extremas figuran en número considerable otros nombres que estuvieron al servicio musical del Papa. La inestabilidad de los mismos y el defecto de listas completas y periódicas no permite fijar las fechas de su ingreso y permanencia en la Corte pontificia. Debido a ello creo conveniente citarlos por orden onomástico dividido en tres secciones.

\section{Cantores pontificios:}

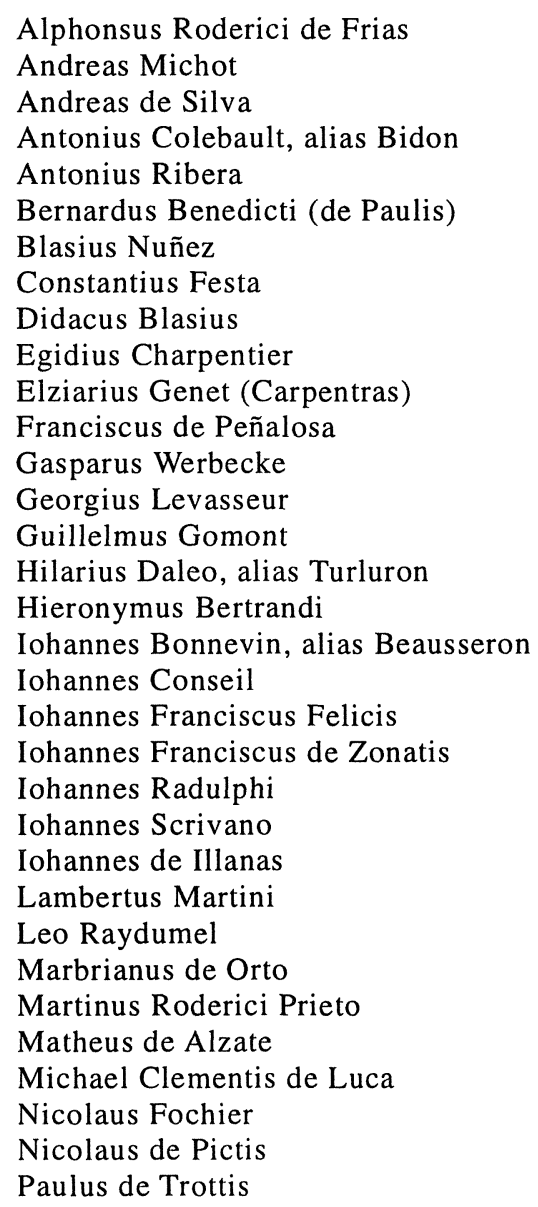


Petrus Jonauld alias Brule

Petrus de Milleville

Petrus Paulus Mastang

Petrus Paulus Remigius

Petrus Perez de Rezola

Petrus Pirrinus

Petrus de Vian

Robertus de Thinille

Thomas de Fazanis

Thomas de Macchia

Vincentius de Misonne

\section{Cantores cortesanos}

Andreās de Silva

Camillo filio Jo. Marie de Medicis

Cesar de Johanne Antonii de Tolentino

Claudius de Alexandris (Alexandrino)

Firminus le Clerc

Franciscus Vanelst

Gabriel de Laude

Georgius de Parma

Hieronimus de Ameria

Hilarius Penet

Iohannes Ambrosius

Iohannes Bonnevin

Iohannes Brugius

Iohannes Esquinus

Iohannes Franciscus de Manfronibus

Iohannes Jacobus de Cigalis

Iohannes Maria Dominici Alemanus (Johannes Maria de Medicis)

Iohannes Manente

Iohannes Mathias

Jacobus Larcintus

Jacobus de Tarvisio

Jacottinus Lebel

Justinus Doro

Laurentius de Bergomotiis

Martinus

Mathias Marilianus

Nicolaus de Albis

Nicolaus Foncher

Petrus Maler

Raphael Lunerius

Raynerius

Siliminus

Simon Malle

Valentinus de la Rue

\section{Niños cantores}

Hilarius Penet

Petrus de Monchiaron 
Johannes Conseil

Petrus Pirrino.

Músicos $^{45}$

Antonio Maria, piffero

Bartholo, fiamengo

Domenico, piffero

Francesco, trombone

Gabriele de Ancona

Gasparo, fiamengo

Georgio, piffero

Guidantonio da Cesena, piffero

Hieronymo da Asti

Iohanni Jacobo, piffero

Iohanni Maria dal Cornetto

Marco Antonio Musier da Bologna, gravicembalo

Melchiore Milanese, piffero

Michele da Verona, piffero

Zaccaria, trombone

\section{En grupo}

Pietro Malet

Francesco Imperato

Guillelmo Chiardo, pifferi

A los cantores y músicos de la Capilla privada del Papa les faltó estabilidad en su cargo. La actuación de muchos de ellos no duró mas de unos pocos meses. Este constante e inaudito fluir de artistas cayó en detrimento de aquella homogeneidad que se requiere en toda ejecución artística de conjunto, y fue, tal vez, la causa de que la música instrumental y vocal profana no alcanzase en ella el mismo grado de desarrollo que la polifónica religiosa.

A este respecto podemos aducir el texto de la carta escrita el 23 de mayo de 1524 por Giovanni Spataro de Bologna a Petro Aron ${ }^{46}$ :

"Son già passati tri anni, et credo che siano più de quattro, che uno M[esser] Laurentio Burgomozo da Mutina, el quale era cantore de la musica secreta de Papa Leone, mei fu dicto che da M[esser] Adriano [Willaert] musico celeberrimo, il quale sta con lo III Duca de Ferrara, haveva mandato uno duo a la Beatitudine de Papa Leone il quale duo finiva in septima et diceva che li cantori di Sua Beatitudine non lo poterno cantare, ma che fu sonato con li violuni, ma non tropo bene".

Y en contraste el Diario de Paride de Grassis que nos refiere el deseo de Isabel de Aragón Sforza, duquesa de Milán, de asistir a la misa pontifical el día en que se celebraba la Dedicación de la Basílica. de San Pedro. Quería la ilustre dama contemplar la magnificencia de los ritos y

45. Spese private di Leone X, Johannes Lazarus de Magistris "Serapica". 46. Bibloteca Vaticana, Vaticano Latino 5318, f. 212. 
escuchar los cantos de aquella tan renombrada Capilla. Sobre el particular escribe textualmente el citado Maestro de ceremonias ${ }^{47}$ :

"Missa per cantores elegans et mirabilis fuit, per comma musicalia quae divina videbantur, ita ut omnes de novo miraremur et stuperemus".

Podríamos añadir que cuando las capillas de Música del Papa León X y del Rey Francisco I de Francia se encontraron en Bologna, diciembre de 1515, los franceses no dejaron de reconocer y estimar la superioridad de los cantores pontificios ${ }^{48}$.

Sin razón, muchos historiadores, ante las considerables sumas con que el Papa estipendiaba a sus músicos y cantores privados, le han tildado de frívolo y negligente en valorar la música sagrada. Es cierto, en efecto, que el Pontífice se gozaba en la audición de selectas composiciones, incluso en actos profanos; el cardenal Ippólito d'Este en una carta a su hermano el duque de Ferrara nos cerciora de ello $^{49}$ :

"Mainente de Reggio cominciò a cantare certi suoi sonetti che aveva fatto in Laude de dicta mogliera del Magnifico [Lucrezia Borgia] neli quali la nominava duquesa de Modena. Et S. Santità disse: perchè non cantate voi qualche altra cosa, hora che sono li di sancti di Pasqua come è la Gloria et Tedeum et lassate stare queste cose de amore? Et cosi Mainente se mise a cantare la Gloria in excelsis". 50

Fabroni, por otra parte, menciona el testimonio de Mateo Herculano, amigo de León X, el cual decía que el Papa estimaba sobremanera las melodías escritas "ad laudem et gloriam Dei",51.

Y Elziario Genet, apodado "il Carpentraso", en una dedicatoria al Cardenal Hipólito de Medicis cuenta de sí mismo que fue enviado por el Rey de Francia Luis XII a la Corte pontificia para llevar la dirección de la Capilla de Música $^{52}$, y añade que, durante su ministerio, pudo constatar la preferencia que el Papa León X dio siempre al repertorio sagrado, en cuya audición,

"ille pias saepe lachrymas hujusmodi concentus eliceret".

Además — prosigue Carpentras - por consejo del mismo Papa, dejé de componer frótolas, para dedicarme exclusivamente a la música sagrada ${ }^{53}$.

Finalmente basta considerar las propias expresiones del Pontífice en sus escritos y documentos. Este es un ejemplo ${ }^{54}$ :

47. Mariano Armellini: Paride de Grassis, Diarium Curiae Romanae, 459.

48. Mémoires du Maréchal de Florange, ed. Goubaux et Lemoine I, 1923, 212.

49. A. FerRajoli: Il ruolo della Corte di Leone X, Roma 1911, 488.

50. Se trata de Giovanni Manente di Reggio Emilia, cantor desde 1 de abril de 1514, camarero personal del Papa con el salario de cincuenta ducados al mes. Archivio Vaticano, Diversorum Cameralium, 631, 239 B.

51. Angelo FABroni: Vita Leonis X, Pisa 1797, 296.

52. J. M. Llorens: "Los maestros de la Capilla Apostólica hasta el pontificado de Sixto V (1585-1590)", Anuario Musical, Barcelona 43, 1988, 35-65.

53. A. Schmid: "Beitrage zur Literatur und Geschichte der Tonkunst", Revue Cäcilia de Deln, XXII. 1844, 203.

54. Archivio Vaticano, Diversorum Cameralium 63, f. 81. 
"Cupientes ut Capella nostra ad laudem eius qui habitat in excelsis, divinis praeconiis valeat resonare ac cantores in ea divina cantantes officia illis majori diligentia insistant”.

El esplendor y brillantez de aquella Corte leonina jamás logró oscurecer ni amortiguar el propio de los Farnese. Es mas, el cetro de monarca del humanismo romano se mantuvo firme en la mano de nuestro cardenal, considerado por Marcantonio Flaminio "Vatum decus et spes unica".

Nadie podía competir con él. Ludovico Ariosto en el último canto de su poema cita nombres tan insignes como eruditos:

\footnotetext{
"Ecco Alessandro, il mio signor Farnese

O dotta compagnia che seco mena!

Fedro, Capella, Porzio, il Bolognese,

Filippo, il Volterrano, il Maddalena,

Blosio, Piero, il Vida cremonese.

D'alta facundia inestimabili vena.

E Lascari e Musuro e Navagero

E Andrea Marone e'l monaco Severo".
}

Según costumbre, los poetas que frecuentaban los palacios de sus mecenas no desperdiciaban ocasión alguna para hacer gala del arte del bien decir en alabanza de los reunidos. Momento oportuno lo encontraron frecuentemente en las horas de solaz al término de una jornada de caza. León X y Paulo III se entregaron periódicamente a este pasatiempo. Iban acompañados de sus cardenales palaciegos, cortesanos, familiares, guardias, músicos, bufones y poetas. Uno de los lugares preferidos por las condiciones que ofrecía era el de la Magliana, a cinco millas de Roma. Sixto IV construyó un palacete que Paulo III adornó con pinturas murales de tema musical. La cena daba oportunidad a músicos y poetas para manifestar sus dotes artísticas. Repetidas veces se señalan en los registros, pagos como estos ${ }^{55}$ :

"Quando andò la música a la Magliana". "Per far fare libri di musica per andar il maggio a la Magliana”.

El Cardenal Farnese que disponía de parajes de abundante venación recibió, en no pocas ocasiones, la visita del Papa. En efecto, en enero de 1514, le acogía con toda pompa en el lindo lugar de Canino, agradable por su dulce clima. Los acompañantes fueron doce cardenales, varios condes, prelados, soldados, músicos y bufones. El poeta de la corte, Baldassare Molosso di Casalmaggiore, conocido también por el nombre de Tranquillo, preceptor de Pierluigi, celebró con versos aquel espléndido gaudeamus, en un poema titulado "Paliettum". Al atardecer, los músicos al son de sus instrumentos, dieron aviso de concentración para hacer la entrada solemne en el castillo con el botín capturado. Durante la opípara cena, en conversación entretenida, se comentaron los sucesos vividos en pleno bosque. Al término de ella, Mario - así llamaban a Grapaldo da Parma- cantó acompañándose él mismo con la cítara, que dejó después lugar a

55. Archivio Di Stato, Roma. Spese private di Leone X. Johannes Lazarus de Magistris. "Serapica" III. f. 2v 
músicas y cantos en dulce armonía. Ni los poetas ni los cronistas son más explícitos en el relato de las composiciones interpretadas.

La cordialidad del Pontífice para con el Cardenal se puso de manifiesto en diversas ocasiones. Una de ellas, gran paso de aproximación al Solio pontificio, fue el cambio de Cardenal diácono a Cardenal obispo de Frascati. Como consecuencia, fue necesario primero, la ordenación sacerdotal, que recibió el 26 de junio de 1519, y la consagración episcopal después, el día de la Visitación de Nuestra Señora. La ceremonia tuvo por marco el suntuoso palacio de la Cancillería. El propio Pontífice actuó de padrino, y le obsequió con un anillo que llevaba engarzada una valiosa piedra. El Diario de Sanuto al referir este acontecimiento informa ${ }^{56}$ :

"L'episcopato Tusculano, per la morte del reverendissimo Camarlengo è stato conferito per il Pontefice al reverendissimo Farnese, primo et antiquo diacono, il qual ha fatto imo una cena al Pontifice et a 20 cardinali ne la quale furono pavoni che costarono sei ducati el paio."

A la muerte del Papa León X, $1^{\circ}$ de diciembre de 1521, Alejandro Farnese era uno de los candidatos con mayores probabilidades de alcanzar el Solio Pontificio. Sanuto ${ }^{57}$ refiere la inquietud que reinaba en el Colegio elector y narra que el Cardenal Julio de Medicis apoyaba la candidatura con estos términos: "Fate Farnese ch'è gentilissimo, nobilissimo, litterato, costumato et degno". Sin embargo, la elección, 9 de enero de 1522, había de recaer en el Cardenal de Tortosa, ausente, Adriano Florensz de Utrecht, que tomó el nombre de Adriano VI.

56. Marino SANUTO: I Diarii XXVII, col. 457, 471. Un precioso retrato del cardenal ostentando el valioso anillo, regalo del papa, obra de Tiziano, se exhibe en la sacristía de la catedral de Toledo.

57. Ibídem, XXXII, f. 357. 